\title{
EchoGéo
}

15 | 2011

La Belgique, État prospère en décomposition au cœur de l'Europe du nord-ouest

\section{Le regard des autres}

Jean-Louis Chaléard

\section{OpenEdition}

Journals

Édition électronique

URL : https://journals.openedition.org/echogeo/12350

DOI : 10.4000/echogeo. 12350

ISSN : 1963-1197

Éditeur

Pôle de recherche pour l'organisation et la diffusion de l'information géographique (CNRS UMR 8586)

Référence électronique

Jean-Louis Chaléard, «Le regard des autres », EchoGéo [En ligne], 15 | 2011, mis en ligne le 11 avril 2011, consulté le 03 août 2021. URL : http://journals.openedition.org/echogeo/12350 ; DOI : https:// doi.org/10.4000/echogeo.12350

Ce document a été généré automatiquement le 3 août 2021.

EchoGéo est mis à disposition selon les termes de la licence Creative Commons Attribution - Pas d'Utilisation Commerciale - Pas de Modification 4.0 International (CC BY-NC-ND) 


\title{
Le regard des autres
}

\author{
Jean-Louis Chaléard
}

1 Cette nouvelle livraison d'Échogéo se place sous le signe d'une double ouverture. D'abord celle de la géographie française à l'étranger. Ensuite, celle des partenaires de la revue, avec la participation de plusieurs collègues belges à ce numéro.

2 La coopération française est largement orientée vers l'Afrique francophone, pour des raisons linguistiques et surtout politiques. Le "pré-carrré » a longtemps été favorisé par les autorités françaises et il était plus facile d'aller travailler en Afrique francophone que dans beaucoup d'autres régions du monde, spécialement quand il s'agissait des pays du Sud. Nombreux sont les géographes de l'hexagone qui ont enseigné dans les universités africaines dès les années 1950-1960. La recherche institutionnelle outre-mer, à travers des organismes comme l'ORSTOM, a longtemps concentré une grande partie de ses forces sur l'Afrique. La coopération avec ce continent a pris aujourd'hui des formes différentes, mais elle reste toujours importante. Cela ne doit pas occulter le fait que la coopération française depuis plusieurs décennies s'est largement ouverte sur d'autres mondes. Un des intérêts du dossier de la rubrique Sur le métier présenté par Alexis Sierra sur la coopération bilatérale et la recherche en partenariat est justement de montrer un autre volet de cette action, en s'appuyant sur des cas pris en Amérique latine. Les collaborations n'y souffrent pas des ambiguïtés et des pesanteurs héritées du passé colonial. Les difficultés sont ailleurs. Moins sans doute dans la langue que dans des histoires et des cultures différentes. Cependant, au-delà des difficultés matérielles et de suivis auprès d'interlocuteurs qui peuvent changer, les exemples de partenariat avec le Brésil et l'Équateur mettent en évidence l'intérêt de ces échanges et les profits mutuels qui peuvent en être tirés. Ils supposent du temps, la mise en place de réseaux sur la durée, la présence des chercheurs et des universitaires sur le terrain, qui permettent l'instauration de relations de confiance entre partenaires. Si les géographes ne sont pas toujours privilégiés dans ces coopérations, ils y trouvent cependant pleinement leur place, par leur réflexion sur l'environnement, sur l'aménagement, sur la gestion des territoires. 
3 L'article Sur le vif de Philippe Pelletier traite aussi de la géographie et de son rôle. Il aborde, conformément à l'orientation de la rubrique, un thème en pleine actualité : le séisme au Japon suivi d'un tsunami et d'un accident nucléaire. C'est l'occasion d'analyser la façon dont les médias traitent parfois l'information, simplifiant ou déformant les phénomènes, abusant des clichés ou de témoignages sensationnalistes. L'auteur cite des propos qui laissent perplexes par leur degré d'approximation. À l'opposé, il essaie d'aller au-delà de l'émoi légitime face au drame japonais, pour proposer un autre discours qui remet en place quelques vérités scientifiques et tente d'analyser l'enchaînement géographique des catastrophes, ainsi que leur contexte socio-culturel lié aux caractéristiques du Japon.

De territoires, de visions parfois simplificatrices d'une réalité complexe, il en est question également avec la Belgique qui occupe une place privilégiée dans ce numéro. Le dossier Sur le champ lui est consacré. Ce pays a eu droit à la une de l'actualité pendant quelque temps en raison de la crise politique qui y perdure et du conflit entre Flamands et Wallons. Le dossier essaie de faire comprendre ces problèmes mais surtout ne se limite pas à ce volet réducteur. Il insiste sur les évolutions du pays depuis plusieurs décennies, comme le montre la comparaison instructive de C. Vandermotten, entre une description géographique de 1954, tirée d'un manuel et la réalité que nous présentent les différents auteurs aujourd'hui. Il montre une autre Belgique que celle que nous renvoient les médias focalisés sur la question fédérale, avec ses dynamismes économiques et diverse dans ses paysages. Le rôle, ou la faiblesse, du pouvoir central face aux pouvoirs locaux y est alors vu sous un autre jour. Et les mises en perspective historiques n'en prennent que plus de sens, inscrites dans le temps long d'une histoire qui nous renvoie, au-delà de l'État créé en 1830, à l'autonomie des cités marchandes du Moyen-Âge et au processus d'une poussée régionaliste émanant aussi bien du nord que du sud depuis près d'un siècle.

5 Un dossier Sur l'image complète la présentation du pays. Les auteurs assument une certaine subjectivité de leurs choix iconographiques. Les clins d'œil à une Belgique de " guides touristiques » ou au "plat pays » de Jacques Brel nous donnent autant à voir qu'à réfléchir sur l'image, sur son apport à la géographie et sur ses limites. Le dossier confirme l'image d'une Belgique de la diversité, plus complexe que le simple clivage Flandres-Wallonie pourrait le laisser croire, et dont les difficultés, selon l'expression de C. Vandermotten, « inquiètent peut-être plus à l'étranger que dans le pays ».

Le compte-rendu de l'ouvrage de Jean-François Bayart par Philippe Gervais-Lambony sur les études postcoloniales renvoie à des questions posées dans les dossiers de ce numéro, même si le thème en est bien évidemment différent. La réflexion sur les études postcoloniales concerne l'ensemble des sciences sociales et donc la géographie. Elle ne peut laisser indifférent ceux qui montent des programmes de coopération internationale, car elle les interroge sur leurs pratiques comme sur le contenu des savoirs qu'ils mobilisent ou diffusent. Elle ne peut laisser indifférent non plus, tous ceux qui s'interrogent sur l'évolution du regard de la recherche sur le monde, sur le poids, dans ce contexte, de la "science occidentale", et sur les conditions de production de la connaissance scientifique. 